\title{
Linx
}

Revue des linguistes de l'université Paris X Nanterre

$8 \mid 1996$

Du dire et du discours

\section{Code écrit, code oral : deux matérialités abordées à travers les constructions segmentées}

\section{Bruno Martinie}

\section{(2) OpenEdition}

\section{Journals}

Édition électronique

URL : http://journals.openedition.org/linx/1152

DOI : 10.4000/linx.1152

ISSN : 2118-9692

\section{Éditeur}

Presses universitaires de Paris Nanterre

\section{Édition imprimée}

Date de publication : 1 septembre 1996

Pagination : 111-135

ISSN : 0246-8743

\section{Référence électronique}

Bruno Martinie, " Code écrit, code oral : deux matérialités abordées à travers les constructions

segmentées », Linx [En ligne], 8| 1996, mis en ligne le 13 juillet 2012, consulté le 19 avril 2019. URL

http://journals.openedition.org/linx/1152 ; DOI : 10.4000/linx.1152

Ce document a été généré automatiquement le 19 avril 2019.

Département de Sciences du langage, Université Paris Ouest 


\title{
Code écrit, code oral : deux matérialités abordées à travers les constructions segmentées
}

\author{
Bruno Martinie
}

\section{Introduction}

1 Le projet initial de cet article était d'examiner de façon "exhaustive" les rapports entre oral et écrit à partir d'un travail sur corpus. Mais la diversité des problèmes rencontrés s'est révélée telle que nous avons dû restreindre le champ d'observation. Pour aller vite, poser que l'oral dispose de propriétés sui generis, cela signifie que les modèles proposant de l'intégrer doivent subir des adaptations. Les implications sont diverses ${ }^{1}$ :

- Méthodologiquement, cela pose la question de la constitution des corpus².

- épistémologiquement, on en vient rapidement à s'interroger sur la mise à l'écart des données orales dans les sciences du langage ${ }^{3}$. Un "oubli" d'autant plus paradoxal que le rejet de l'écrit, dépeint comme un « travestissement de la langue » dès le CLG (pp. 51-52)4, se trouve d'une certaine façon à la source du fondement de la linguistique, dont la scientificité s'est érigée sur la base du phonème, et non de la lettre ${ }^{5}$.

- L'omniprésence de la dimension sociolinguistique ne peut être négligée, puisque chacun est conscient que l'oral, plus que l'écrit, est enclin à présenter une hétérogénéité qui a de toute évidence déterminé sa mise à l'index de la description grammaticale ${ }^{6}$.

- Enfin, sur le plan théorique, la prise en compte de l'oral fait figure de gageure : sa " profusion $»^{7}$ laisse poindre, du point de vue descriptif, le risque de ne faire que des « collections de papillons » (Chomsky, 1977, 75), face à des théories s'exposant à « bâtir sur du sable » (Deulofeu, 1984, 58). Nourri de données “authentiques" sans lesquelles il apparaît exsangue, le descriptivisme linguistique se heurte à de nouveaux obstacles : entre autres, si écrit et oral disposent de spécificités propres en nombre suffisamment important, à partir de quand faut-il poser l'existence de deux systèmes différents ${ }^{8}$ ? 
2 En définitive, l'oral en tant qu'objet légitime d'observation linguistique peut difficilement faire l'économie d'une réflexion sur la matérialité de l'écrit, avec les interférences idéologiques (normativisme, purisme) qui affleurent inexorablement.

3 L'entreprise originale de Halliday nous servira ici de point de départ. Selon lui, oral et écrit présenteraient des niveaux de complexité différents : complexité grammaticale pour l'oral, complexité lexicale pour l'écrit (1990, chap. V et VI). Or, outre la procédure technique qu'elle sollicite ${ }^{9}$, une telle position suppose qu'on puisse confronter des données équivalentes, proportionnelles. C'est dans ce but que nous avons mis en place une méthodologie que nous exposons à présent.

\section{Formes segmentées et travail de "collation"}

\section{Le matériau}

4 Le face-à-face de la présidentielle du 2 mai 1995 opposant les candidats Jacques Chirac et Lionel Jospin nous a fourni les données de travail. Nous avons procédé à une transcription orthographique aménagée (désormais TOA) portant sur l'intégralité du débat (durée : 2 heures $)^{10}$. Les données obtenues ont ensuite été confrontées avec celles fournies par le "texte intégral" figurant dès le lendemain dans deux quotidiens de la presse nationale : Le Figaro et Libération ${ }^{11}$.

\section{Hypothèse sociolinguistique}

5 Notre hypothèse de départ revêt un caractère axiomatique: en toute logique, les transcriptions des deux quotidiens ont pour simple but de restituer le contenu de la prestation de chaque candidat. Elles différent en cela de TOA, issue d'une procédure systématique, et dont on admettra par commodité qu'elle est l'image fidèle des productions orales ${ }^{12}$. Par ailleurs, les transcriptions de la presse ont été faites dans l'urgence $^{13}$. Elles sont donc susceptibles de faire ressortir une certaine spontanéité de la part des scripteurs, et par conséquent :

- D'une part, des réflexes normatifs et idéologiques d'autant plus probables qu'il s'agit de rapporter des paroles de locuteurs présidentiables ${ }^{14}$.

- D'autre part, des réflexes liés au décalage entre pratique orale et pratique écrite.

6 Nous proposons de tester cette hypothèse à travers un travail de "collation", en misant que les écarts perceptibles entre TOA et les transcriptions de la presse sont un lieu d'observation pertinent dans une problématique de description de l'oral.

\section{Choix du phénomène observé : les tours segmentés}

7 Ce type de construction nous semble présenter les caractéristiques requises pour l'objectif poursuivi :

- Le corpus TOA nous en fournit un nombre satisfaisant, de formes variées ${ }^{15}$.

- On sait que les constructions en question font l'objet de jugements normatifs dans les grammaires, bien qu'on reconnaisse leur pérennité ${ }^{16}$ : Grevisse remarque qu'elles « étaient assez courantes dans l'ancienne langue écrite, moins soucieuse de logique et plus proche du style 
parlé $[. .] ».(1993, \S 228$, nous soulignons). Blanche-Benveniste et Jeanjean $(1987,26)$ signalent leur présence dans le Roman de la Rose.

- Enfin, tous les tours segmentés ne sont pas également condamnés : Berrendonner et Reichler-Béguelin $(1989,118)$ parlent de « dérogations » sous certaines conditions que nous tenterons d'examiner plus loin.

Fréquence d'apparition du phénomène, diversité des formes qu'il revêt, prestige/ condamnation dont il fait l'objet : autant de propriétés permettant une approche telle que celle que nous proposons ici.

\section{Segmentations accompagnées de reprise}

\section{Les formes en moi je}

Le corpus TOA livre un total de 46 occurrences ${ }^{17}$ de type :

(1) // (r) moi <j'ai pris simplement le journal officiel > //

Curieusement, on constate une étonnante stabilité de ces tours sur la personne : aucun lui $i l$, nous nous ou nous on par exemple. Seule exception :

(2) /// (r) ${ }_{L U I}</ /$ pense < /// et il l'a dit < // qu'au fond $</ /$ (r) il y a $</$ une sorte $<$

/ de DÉrive $<$ / monarchiste $>$ //

avec la forme disjointe en tête, mais ne donnant pas lieu à la reprise en il. Outre que cette ressource n'est pas disponible sur la première personne, on peut supposer que la situation d'énonciation a en partie déterminé cette régularité, étant entendu que pour les deux protagonistes de l'échange, il s'agissait de présenter un programme par opposition à celui de l'autre candidat. D'où, par exemple, la surreprésentation des moi je dans des constructions à verbe d'opinion du type :

(3) /// (r) moi je crois < / qu'il faut d'abord < // rééquilibrer les institutions > /

11 Or, l'effet de contraste produit par ce type de dispositif ${ }^{18}$ répond particulièrement bien à ce besoin énonciatif. A l'inverse, dans :

(4) (...) je suis ${ }^{\wedge}$ heureux < / de faire ce débat $>/ / /$ (r) euh ça n'a pas \# été possible au premier tour $</ / /$ euh nous le faisons maintenant $</ /$

l'énonciation semble contraindre négativement la possibilité de nous nous ou de nous on, étant donné la visée argumentative du locuteur ${ }^{19}$.

Quoi qu'il en soit, et conformément à nos attentes, les deux journaux n'offrent aucun cas de moi je absent du corpus TOA. Mais plus surprenant est le nombre de moi je restitués tels quels :

- Le Figaro présente un indice de restitution supérieur à 80\% des occurrences (37/46).

- Ce même indice s'élève à $74 \%$ (34/46) pour Libération.

Reste donc un certain nombre de cas de moi non restitués. Parmi ceux-là, l'exemple de

(5) /// (r) vous savez moi ça aussi < / c'est $^{\wedge}$ une chose que j'ai bien observée < /

est particulièrement intéressant : le pronom de reprise (je) rentre dans une phrase clivée, elle-même disposée dans une construction à présentatif dont l'objet est doublement marqué (ça $\left.c^{\prime}\right)$. Un "seuil de tolérance" aurait-il été franchi au nom d'une limite fixée en matière de "redondance $»^{20}$ ? Le fait que le moi ait disparu des deux transcriptions semble corroborer cette hypothèse.

Nous nous sommes également posé la question de savoir ce qui avait pu conditionner le maintien de moi. Un fait retiendra en premier lieu notre attention : la forte fréquence de 
connecteurs $^{21}$ devant les segments moi je. Or, la tolérance à la segmentation paraît corrélée à la présence de cette classe d'éléments, si l'on tient compte du fait que sur les 13 occurrences de ce type relevées dans le corpus TOA, aucun moi n'a été supprimé ${ }^{22}$. La solidarité du dispositif contrastif CONNECTEUR + moi je est confortée par deux exemples :

(6) mais moi $</$ je propose $</$ de faire un vrai $</$ service républicain $</$

(7) alors moi </je trouve < / qu'il y a tout de même là < / une certaine continuité < /

où le Figaro et Libération optent pour deux transcriptions différentes : le dispositif est soit entièrement supprimé, soit intégralement restitué.

(6a) Mais moi, je propose de faire un vrai service républicain [...]. (Figaro)

(6b) Je propose un vrai service républicain. (Libération)

(7a) Alors moi,je trouve qu'il y a tout de même là une certaine continuité. (Figaro)

(7b) Je trouve qu'il y a là, tout de même, une certaine continuité [...]. (Libération)

L'idée a été régulièrement défendue qu'au-delà des moyens purement syntaxiques mis en œuvre dans les phrases segmentées, il existerait un conditionnement discursif ${ }^{23}$. Plus précisément, les relations "d'affinité" entretenues entre la segmentation et la coordination ont souvent été relevées. Curieusement, cela se fait :

- Soit en terme d'opposition : c'est le cas de Guilhaumou et Maldidier (1983) pour qui « [...] la thématisation focalise du préconstruit $[. .$.$] » (112) là où « [...] la coordination [...] construit$ par un effet de globalisation de nouveaux référents » (ibid. $)^{24}$.

- Soit en terme de complémentarité, comme chez Ruppli (1991) qui constate que certains cas de mise en relief « [...] renforcent la liaison coordination [...] entre deux phrases » $(50)^{25}$.

Dans les cas où le disloqué est introduit par un connecteur, nos données semblent rejoindre la deuxième position : la " pression thématique » (cf. Ruppli, op. cit. 51) propre à ce type d'énonciation aurait donc conditionné le maintien de CONNECTEUR + moi je dans les deux journaux étudiés. Ce dispositif offre un certain nombre de ressources discursives, notamment la possibilité d'introduire des thèmes contrastés sur la première personne. C'est ainsi que dans :

(8) / un député > / un ministre < // euh qui n'est pas < / qui n'a pas de mandat local $>$ / qui n'a pas l'obligation < / d'aller euh passer ses week-end $</$ sur le TE rrain $<>$ / sur les CHAmps de foire $<>/$ dans les quartiers difficiles $<>/ /(r)$ auprès des gens $<>/ / /(\mathrm{r})$ et bien: / c'est ${ }^{\wedge}$ un très grand danger qu'il devienne rapidement ${ }^{\wedge}$ un apparatchik >> //(r) et moi</ je ne: suis pas favorable du tout > / à cette: / à cette: solution $>>/ /$

on peut difficilement désolidariser les deux termes du dispositif en question. En effet, la présence $\mathrm{du}$ coordonnant seul transférerait le contraste portant initialement sur l'énonciation vers la phrase coordonnée dans son intégralité: c'est ainsi que le fonctionnement des "coordonnants" dans le dispositif ici mis en jeu semble dépasser le rôle grammatical qu'on leur assigne traditionnellement.

\section{Autres formes avec reprise}

Dans tous les exemples précédents, la présence de moi impose la réalisation de la forme conjointe correspondante (je), du fait que cette dernière est le sujet construit par le verbe situé à droite. Si ce sujet est un SN, comme c'est le cas dans l'exemple :

(9) $m o i</ m a$ vision < // est TRÈs différente $</ /$ (corpus TOA)

(9a) $*$ moi $</$ la vision $</ /$ est TRÈs différente $</ /$

(9b) $*$ moi $</$ une vision $</ /$ est TRÈs différente $</ /$ 
la forme du déterminant est contrainte, ainsi que le montrent $(9 a+b)^{26}$. Comme précédemment, deux traitements étaient possibles pour (9) : maintien de la séquence telle quelle (Figaro), ou suppression du moi (Libération).

Qu'en est-il des autres formes segmentées repérables en TOA ? Conformément à l'objectif de départ, nous tenterons de défendre l'idée que les transcriptions des deux quotidiens examinés ont suivi une logique à travers laquelle il est loisible d'observer les contraintes pesant sur les constructions segmentées. De quelle nature sont ces contraintes? Nous proposerons des pistes d'analyse susceptibles d'éclairer ce point.

\section{SN Indéfini + reprise (1 occurrence)}

Le seul exemplaire du corpus, à savoir :

(10) /// (r) un petit entrepreneur $>/$ aujourd'hui $</ /$ il est ${ }^{\wedge}$ incapable $</$ d'avoir euh $/ /$ de constituer ses fonds propres $>/ / /$ a, contre toute attente, été restitué tel quel dans les deux quotidiens.

Dans la littérature, il est fréquemment fait mention de l'agrammaticalité de l'indéfini dans les tours segmentés. Diverses pistes sont explorées: la possibilité d'une interprétation spécifique et d'une autonomie référentielle (cf. par exemple Cadiot, 1988, 11) ; le critère de disponibilité en " mémoire discursive » du référent du SN de tête est mis en avant par Berrendonner et Reichler-Béguelin (1989) ; enfin, l'idée que le référent du lexique disloqué doit être identifiable est particulièrement répandue, et se trouve discutée chez de Fornel (1988).

L'examen de corpus est toujours l'occasion de constater la productivité d'une construction dans l'usage d'une période de la langue, et nous conduit en droit à réviser nos jugements d'agrammaticalité. Or, la présence de (10) dans le corpus contredit d'autant plus nos attentes qu'il a été restitué tel quel dans les deux quotidiens. En définitive, la valeur générique de un accompagné d'un présent intemporel semble lever le problème d'acceptabilité en question. On peut le vérifier en introduisant le trait [ ACCOMPLI] :

(10a) * un petit entrepreneur / il a été incapable de constituer ses fonds propres

À l'opposé, l'acceptabilité ne pose aucun problème si le pronom de reprise est "neutre", ( $c^{\prime}$ ou ça). Ainsi, l'exemple suivant :

Par bonheur, une tête de vipère, $c$ 'est triangulaire est mis en avant par Grevisse (\$367) - le seul cas de figure où, à notre connaissance, l'auteur du Bon usage reconnaisse la possibilité de la présence de un dans de telles constructions.

\section{SN défini + reprise (9 occurrences)}

(11) la protestation < /// naturellement \# </ elle s'est $^{\wedge}$ exprimée partout >> ///

(12) le contrat que je propose $</ c^{\prime}$ est $^{\wedge}$ un contrat à durée $</$ indéterminée $>/ /$

(13) les prêts d'accession à la propriété < / vous pouvez toujours les augmenter <

(14) le maintien des banques nationalisées < / ça c'est un système socialiste > /

(15) la règle < / c'était un mètre carré de logement < / pour deux mètres de bureau >

//

(16) la moyenne des grandes villes de france $</$ paris inclus $</$ c'est un tiers (chev.) 
(17) euh: je voudrais dire simplement très vite $</$ alors un mot $</$ sur les deux aut res questions $>/ /$ le service militaire $<j$ 'ai: euh toujours (hein, un) considéré $</ / /(r)$ qu'il était maintenant $</$ tel qu'il est $<</$ dépassé $>/ /$

(18) le service militaire < / c'était l'égalité < / et la liberté $>/ /$

(19) la réduction < / de cette fracture < / C'EST <// LE problème < / essentiel >> ///

\section{"dépersonnification" ou de neutralisation tels que :}

(20) Les filles / C'est beau tant que ça peut / C'est beau comme l'adieu / Et c'est beaucoup mieux (Jacques Brel)

(21) les filles / ils sont mal vus (oral spontané)

puisqu'aucun des SN de tête ne présente le trait [PERSONNEL]. L'absence de tels phénomènes étiquetés "populaires" explique peut-être la restitution quasi automatique des SN définis + reprise dans les deux journaux :

- Parmi les pseudo-clivées, une seule a subi un remaniement. Il s'agit de (14), dont

le ça a disparu d'une des deux transcriptions :

(14a) Le maintien des banques nationalisées, c'est un système socialiste (Libération) ce qui semble corroborer l'idée avancée à propos de (5) faisant état d'une faible tolérance aux cumuls de reprises.

- Dans les trois autres séquences (11, 13 et 17), on remarque que seule (11) permet la suppression de la forme de rappel (le SN de tête occupant la fonction sujet). D'où la transcription du Figaro:

(11a) La protestation, naturellement, s'est exprimée partout [...].

En revanche, le rétablissement d'une forme non segmentée en (13) et (17) s'avère plus délicat : en (13), les prêts d'accession à la propriété occupe la fonction d'objet à droite, et la seule suppression de la forme de reprise ne restituerait pas l'ordre canonique; en (16), l'insertion modalisante j'ai toujours considéré impose la forme de reprise dans la complétive enchâssée sous considérer. La restitution d'une phrase non segmentée ne pouvait se faire qu'au prix d'autres transformations: soit la suppression de la modalisation et de la conjonction, avec mise au présent de l'auxiliaire, soit le déplacement du SN dans la complétive.

\section{SN Démonstratif + reprise (7 occurrences)}

Dans cette catégorie figurent également les cas où le disloqué s'identifie à ça :

(22) ça < / seuls les techniciens < / peuvent nous le dire > //

(23) cet esprit de conquête </ qui nous a marqul longtemps $</$ eh bien $</$ nous ne le retrouverons pas $>/ / /$

(24) $/ / /$ (r) mais // j'en reviens > // tous ces jeunes $>/ /$ on va en former bientôt $</$ deux cent cinquante mille par an $>/$

(25) cette démarche $</ /$ elle est décisive $</ /$

(26) $c ̧ a</ c^{\prime}$ est $^{\wedge}$ une réflexion de bon sens $>/ /$

(27) $c ̧ a</ c^{\prime}$ est $^{\wedge}$ euh: $/ / /$ je le crains $</$ prendre un peu ses désirs $</$ pour des réalités $>>$ /

(28) $c ̧ a</$ ça me choque $>/ /$ 
parmi lesquels ont été relevées 6 modifications.

Jusqu'ici, nous avons évoqué deux contraintes susceptibles de peser sur la restitution/ non-restitution des tours segmentés dans les deux journaux étudiés :

- Une contrainte (A), relative à la fonction de la forme de reprise dans le noyau verbal, celle-ci n'étant supprimable que dans les cas où le disloqué est relié à la fonction sujet, dans la mesure où ce type de segmentation fait apparaitre un disloqué dans une position fonctionnelle, contrairement à ce qui se produit pour l'objet par exemple.

- Une contrainte (B), qui rend compte de la faible tolérance des cas de cumul de reprises (exemples 5 et 14).

Bien que (A) et (B) demandent à être affinées (cf. infra), elles semblent pertinentes pour rendre compte des exemples de cette rubrique :

- Dans les exemples (22), (23) et (24), le disloqué est relié à la fonction objet: conformément à (A), ils n'ont été modifiés dans aucun des deux journaux.

- La tendance s'inverse pour la fonction sujet (exemples de 25 à 28). Le Figaro ne restitue pour sa part aucune segmentation: le elle de (25) et les ça de tête en (26), (27) et (28) ont tous été supprimés (à noter qu'en 27, la réorganisation porte sur toute la séquence, transformée en Il ne faut pas prendre ses désirs pour des réalités).

- Libération restitue deux occurrences sur les quatre, mais (A) n'est pas automatique sur la fonction sujet (exemples 10 et 11). Il s'agit de (25) et (27) avec, dans le deuxième cas, la présence de cela au lieu du ça initial ${ }^{28}$.

La contrainte (B) ne peut être ici vérifiée, puisque le corpus ne fournit pas d'exemple du type

(25a) Cette démarche, ça c'est décisif parallèlement à ce qu'on avait en (14) sur le déterminant défini. En revanche, au regard de la faible tolérance aux ça $+c^{\prime}$, on peut faire les observations suivantes, qu'il serait toutefois nécessaire de vérifier sur un plus grand nombre d'exemples :

1. La contiguïé de deux pronoms dans une construction segmentée est relativement bien perçue lorsque ceux-ci sont de forme différente (cf. les formes en moi je).

2. Tel n'est pas le cas lorsqu'il s'agit de deux formes identiques ou proches (ça $c ̧ ̧ a$ ou $\left.c ̧ a+c^{\prime}\right)$. Dans ce cas, la norme appliquerait une règle d'haplologie pronominale, qui se trouve par ailleurs appliquée dans certains cas (succession de deux en par exemple).

3. La tendance (1) n'est plus vérifiée dans les cas où les deux pronoms contigus sont reliés à deux places de rection différente (exemple 5), la syntaxe se présentant alors de façon plus "éparpillée".

\section{Circonstant + reprise (5 occurrences)}

(29) et quant au referendum $>/ /$ suR la question du quinquennat $</ /$ (r) il sera fait $<>$ / vite $</ /$ (corpus TOA)

(30) moi je pense < / que s'agissant < / de la politique de l'emploi </// (r) elle a été > /// depuis toujours < /// (r) considérée en réalité < / comme: /// devant: // régler < /// un problème $</ /$

(31) en ce qui concerne les salaires < /// (r) d'abord il y a nécessité < / de les augmenter dans leur $</ /$ dans le principe $>/$

(32) LES nominations $>/$ et là $</ /$ je réagis à des choses $</$ qui ont étés dites $</$ par monsieur chirac $</ /(r)$ bien que j'aurai // (r) D'Autres choses à dire $</$ touchant ma conception $>/ /(\mathrm{r})$ sur les nominations $>/ / /(\mathrm{r}) \mathrm{moi}</$ je suis pas du tout hostile à ce qu'on réduise < / le nombre des nominations euh: faites en conseil des ministres > // 
(33) en ce qui concerne donc < / le crédit < / par exemple < // moi je suis favorable < / à

ce qu'on revienne < à une certaine sélectivité $d u$ crédit < //

\section{suivante :}

1. Les nominations $=$ Constituant lexical détaché

2. et là $[. .$.$] conception =$ incise

3. sur les deux nominations $=\mathrm{SP}$ détaché

4. moi $[. .$.$] ministres =$ noyau verbal

(34) Lorsqu'il est arrivé, il a pris la parole (avec coréférence des constituants soulignés). apposé. sujet, à l'instar de (30), contrastant avec (35) ${ }^{29}$.

(35) ?? Arrivé au premier étage, il y avait un maître d'hôtel près de Marc phénomène de segmentation tel qu'il est envisagé ici fait systématiquement apparaître le surmarquage d'une des places de rection d'un verbe constructeur (en 34, les deux occurrences de il disposent chacune d'un verbe conjugué). Aussi les circonstants détachés de cette rubrique sont-ils des SP, à l'exception de (30) faisant apparaître un participe sont diverses : sujet (29 et 30 ), objet (31), et génitif dans le cas (33) où la reprise ne s'effectue toutefois pas par une proforme ("autoreprise").

a longueur de l'incise a donné lieu à un retour en arrière ${ }^{30}$, ce qui aboutit à la reformulation de (1) en (3). Le détachement apparaît ainsi une fois sous la forme d'un SN, puis sous la forme d'un SP, avant d'occuper la fonction d'objet dans le noyau verbal.

L'hypothèse concernant le seuil de tolérance limité à deux réalisations d'un même élément de rection se vérifie encore dans ce cas, et rend compte de la transcription des deux journaux, qui ont toutefois opté pour deux solutions différentes :

(32a) Sur les nominations: moi, je ne suis pas du tout hostile à ce qu'on réduise le nombre des nominations faites en Conseil des ministres. (Figaro)

(32b) Les nominations, et là je réagis à des choses qui ont été dites par Monsieur Chirac bien que j'aurai d'autres choses à dire touchant ma conception sur les 
nominations, je ne suis pas du tout hostile à ce que l'on réduise le nombre des

nominations faites en Conseil des ministres. (Libération) non conforme à ce qu'indique la prosodie indiquée en $(32)^{31}$, mais la transcription de Libération permet de faire de sur les nominations un élément de rection du verbe de l'incise. En définitive, dans les deux journaux, le triple marquage et le retour en arrière sont ainsi évités.

\section{Formes sans reprise}

Nous nous pencherons pour finir sur les cas où le disloqué de tête n'est pas repris dans la séquence qui suit. Nous avons exclu les cas de circonstants du type :

(36) en ce qui concerne euh: l'évolution euh / de ce système constitutionnel $>/ /$ moi < / j'ai

un point de vue Différent $<$ / (corpus TOA)

jouissant d'une grande mobilité, dans la mesure où ils portent sur l'ensemble de la phrase. Leur positionnement en tête sans reprise ne pose par conséquent aucun problème d'acceptabilité.

40 l'élément présenté en tête ne fait l'objet d'aucune reprise dans le noyau verbal. Il s'agit d'énoncés du type :

(37) ces souliers / j'écrase les pieds de tout le monde (Deulofeu, 1981)

où la relation pressentie entre le $\mathrm{SN}$ de tête et le reste de l'énoncé n'est pas de nature morphosyntaxique. On est dans le domaine des « compléments ambiants locutoires » dont parlaient Damourette et Pichon pour décrire un niveau de relation syntaxique "lâche » (1911-1930, \$107 et \$110).

41 La présence d'un relateur (préposition ou locution prépositionnelle) introduisant l'élément de tête semble de nature à restituer un énoncé "standard" (pour 37, avec introduisant ces souliers) et donc à tempérer le jugement normatif. À cet égard, le traitement de l'objet proposé par Grevisse est assez parlant :

(a) Lorsqu'il est placé en tête, «[...] le complément d'objet direct est généralement repris par un pronom personnel devant le verbe » (\$296) :

(38) Des yeux de statue, on en avait vu par milliers. (P. Loti)

En l'absence de reprise, le tour est jugé « [...] d'une langue assez relâchée » (ibid., cf. aussi \$367b). Parmi les exemples cités :

(39) Un grand troupeau de pleurnicheurs ils formaient. (Céline)

(b) L'objet indirect est donné comme plus mobile. Aussi son positionnement en tête sans reprise est «[...] tout à fait reçu » (\$367b) : «Dans l'usage ordinaire, on ne reprend pas le complément [d'objet indirect] par un pronom personnel » $\left(\$ 2962^{\circ}\right)$. Parmi les exemples cités :

(40) Aux dames "brûlées" de cette société Odette ressemblait trop. (Proust)

(b') Toujours concernant l'objet indirect, l'auteur du Bon usage donne la reprise comme « obligatoire » $\left(\$ 2962^{\circ}\right)$ en l'absence de préposition en tête :

(41) Cette loi sainte, il faut s'y conformer. (Hugo)

Les limites imposées au mécanisme de la reprise apparaissent pris dans une logique très nette : la forme de rappel est de mise pour souligner la présence de l'objet direct en 
position non canonique (situation a). Elle est également obligatoire pour marquer le statut grammatical de la relation unissant l'OI au noyau verbal dans le seul cas où le trait [ TRANSITIF INDIRECT] n'est pas signalé en tête du détachement (alternative b ou b'). Un cadre logique de la grammaticalité au-delà duquel on se trouvera soit dans le domaine « ambiant » évoqué plus haut, avec, par exemple :

(41a) Cette loi sainte, il faut se conformer soit dans le domaine de la « redondance expressive ${ }^{32}$, dans le cas de :

(41b) A cette loi sainte, il faut s'y conformer

C'est donc dans cet espace (absence de relateur + absence de représentation dans le noyau verbal) que nous observerons les détachements sans reprise. Selon ce double critère, le corpus TOA ne nous fournit que deux occurrences. Hasard du corpus? Il nous parait plus pertinent de voir dans cette sous-représentation la confirmation du faible prestige affecté aux formes sans reprise, compte tenu du registre "soigné" supposé chez les deux présidentiables. Or, la transcription adoptée par les deux journaux vient corroborer ce point de vue. Le cas de

(42) et: euh: / ceux-là < // il faut ${ }^{\wedge}$ un accompagnement > // est particulièrement parlant. D'une part, il répond bien au principe de non-reprise évoqué plus haut: ceux-là n'est introduit par aucun relateur (étant entendu que les coordonnants n'induisent aucune relation de dépendance). D'autre part, le lien qui relie ceux-là au segment de droite n'est pas de nature morphosyntaxique, mais prosodique ${ }^{33}$. En toute logique, il s'agit d'un procédé peu envisageable à l'écrit ${ }^{34}$, ce que confirment les transcriptions du Figaro et de Libération :

(42a) [...] et à ceux-là, il faut un accompagnement. (Figaro)

(42b) [...] et pour ceux-là il faut un accompagnement, [...] (Libération)

Même si les deux prépositions ne sont pas identiques, il nous semble significatif que les deux scriptions confient au marquage morphosyntaxique une structuration assurée par la prosodie dans l'énoncé initial. La confrontation des données telles que nous l'envisageons ici nous semble propre à vérifier dans les faits l'idée généralement admise qu'oral et écrit privilégient respectivement la parataxe et l'hypotaxe, sans qu'il soit possible pour autant d'assimiler hypotaxe et intégration syntaxique ${ }^{35}$.

Par collation, il semble donc loisible d'observer l'esprit de la norme, et de confirmer de facto la position de Berrendonner pour qui «[...] la grammaire normative tend [...] à généraliser à toute configuration syntagmatique le modèle qui vaut pour la proposition, donc à hypertrophier la microsyntaxe aux dépens de la macrosyntaxe» $(1990,32)$. Parallèlement à cela, le cas des nominativus pendens (SN suspendus), à quoi on peut assimiler (42), prend place dans un faisceau de phénomènes ayant récemment conduit à la nécessité du passage à une syntaxe dépassant le cadre purement rectionnel ${ }^{36}$.

On trouve également chez Deulofeu (1982) l'idée que ce seraient des "préjugés logicistes » qui auraient déterminé la mise à l'index des tours comme (42), jugés imprécis et ambigus. S'appuyant sur le fait que l'usage des écrivains du XVIIe siècle reflète sur ce point l'usage "populaire» contemporain ${ }^{37}$, il pose l'existence d'un sous-système permettant de couvrir «[...] de façon "vague" l'ensemble des rections possibles » (p. 64), avec notamment un "marquage zéro" (ibid.) où la relation grammaticale n'est pas précisée.

51 Reste un second cas de détachement sans reprise fourni par TOA : 
(43) l'état impartial < // moi je souhaiterais < // monsieur chirac < // (r) que: // vous: // REgardiez ce que j'ai fait quand j'ai été Ministre de l'éducation nationale < // compte de ce qui se produit dans l'énoncé suivant :

(45) // (r) sur les nominations > /// ( r) moi < / je suis pas du tout hostile à ce qu'on réduise < / le nombre des nominations euh: faites en conseil des ministres > //

(45a) Sur les nominations: moi, je ne suis pas du tout hostile à ce qu'on réduise le nombre des nominations faites en Conseil des ministres.

la finale basse affectant sur les nominations marquant son autonomie, d'où son fonctionnement analogue à celui d'un énoncé sans verbe de type "titre de journal". En définitive, cette disposition semblait toute désignée pour les formes ne présentant ni reprise, ni relateur (43a), à moins qu'on n'introduise un relateur de façon artificielle (42 a $+b)$. Plus encore, la disposition par effet de titre, toujours disponible au transcripteur, a été étendue à certaines formes avec reprise (45a), y compris lorsque ce choix s'avère intenable (44a). 


\section{Conclusion}

La diversité des pistes explorées (pistes énonciative, grammaticale, "macrosyntaxique" et prosodique) nous a certes fait prendre le risque de l'hétérogénéité descriptive. Gageons que cela offre quelques contreparties. Ainsi, on a pu observer que :

- D'une part, rien ne nous autorise à penser que l'oral est plus "expressif" que l'écrit. Plus vraisemblablement, cette caractéristique lui est extrinsèque. Certains dispositifs à segmentation semblent avoir une réalité essentiellement énonciative : que ceux-ci aient été majoritairement restitués montre, en droit, leur transposabilité à l'écrit. D'aucuns verraient sans doute, dans les quelques écarts constatés entre TOA et les transcriptions de la presse, une conséquence des rôles assignés à l'oral et à l'écrit dans nos sociétés ${ }^{41}$. De notre point de vue, un fait nous semble important : la variation n'est certes pas l'apanage de l'oral, mais ce dernier nous en permet l'accès immédiat, même lorsqu'on a affaire à des productions

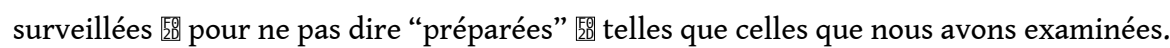

- D'autre part, en entreprenant cette étude, nous nous attendions à une moins grande "fidélité" de la part des transcripteurs par rapport au phénomène observé. L'hypothèse de départ reste cependant partiellement validée : en effet, les modifications relevées dans la presse vont exclusivement dans le sens de la "désegmentation", mais surtout, elles nous semblent révélatrices à au moins deux égards :

1. La disparition des cumuls de segmentation + reprise dans un même énoncé nous permet de rejoindre des positions comme celle de Halliday $(1990,62)$, pour qui l'oral présente une syntaxe plus « clairsemée » (sparse) et « complexe » (intricate) que celle que nous livre l'écrit, ce qu'illustre parfaitement la syntaxe de (5) et le traitement qui lui a été réservé par les deux journaux.

2. L'exploitation de la structuration par voie intonative dans les productions orales, particulièrement saillante dans les cas de non-reprise, ne fait pas réellement partie de la pratique écrite ${ }^{42}$ qui, en tout état de cause, lui préfère un marquage morphosyntaxique.

Parallèlement aux faits de structure auxquels elles nous donnent accès, les données orales posent clairement le problème de la norme linguistique. La délimitation de ces deux ordres est essentielle, ne serait-ce que pour garantir la validité de la description : être conscient d'observer le système, ou seulement l'actualisation d'une norme ${ }^{43}$. D'où, entre autres, la nécessité d'en passer par un examen des pratiques orale et écrite, compte tenu du fait qu'une ressource peut être liée à une matérialité spécifique (comme semblent l'illustrer les configurations intonatives) : nous espérons que l'approche "par collation" proposée ici permet d'aller dans cette direction.

\section{BIBLIOGRAPHIE}

ASHBY, W. J. (1994) An acoustic profile of right-dislocations in French, Journal of French Language Studies, 2, Vol. 4, 127-146. 
BALLY, Ch. (1965) Linguistique générale et linguistique française, (éd. revue et corrigée, 1932 pour la 1ère éd.), Bern, Francke.

BARTHES, R. (1981) Le grain de la voix, Paris, Le Seuil.

BERGOUNIOUX, G., BLANCHE-BENVENISTE, Cl., CHEVALIER, J.-Cl., DUMONT, C., ENCREVÉ, P., PERDUE, C., SIMONI-AUREMBOU, M.-R. (1992) Actualité de l'enquête et des études sur l'oral, Langue française, 93, 94-119.

BERRENDONNER, A. (1990) Pour une macro-syntaxe, Travaux de linguistique, 21, 25-36.

BERRENDONNER, A. et REICHLER-BÉGUELIN, M. (1989) Décalages : les niveaux de l'analyse linguistique, Langue française, 81, 99-125.

BLANCHE-BENVENISTE, C. (1983) L'importance du français parlé pour la description du français tout court, Recherches sur le français parlé, 5, 191-210.

BLANCHE-BENVENISTE, Cl. et alii. (1990) Le français parlé - Études grammaticales, Chapitre II «Macro-syntaxe », Paris, CNRS.

BLANCHE-BENVENISTE, Cl. et JEANJEAN, C. (1987) Le français parlé, CNRS-INALF, Didier-Érudition. BONNARD, H. (1971-1978) article « Anacoluthe » in Le grand Larousse de la langue française, 162-163, Paris : Larousse.

BOSREDON, B. et, TAMBA, I. I. (1992) Thème et titre de presse / les formules bisegmentales articulées par un "deux-points", L'Information grammaticale, 54, 36-44.

CADIOT, P. (1988) Le thème comme synecdoque, Langue Française, 78, 9-25.

CHERVEL, A. (1983) La langue parlée au XIXe siècle, Recherches sur le français parlé, 5, 164-175.

CHOMSKY, N. (1977) Dialogues avec Mitsou Ronat, Paris, Flammarion (coll. Dialogues).

CLÉMENT, D. (1991) Réflexions sur la notion d'intégration en syntaxe ou : qu'entend-on par l'expression X est plus ou moins "intégrée" à la phrase ?, Langages, 104, 7-21.

COMBETTES, B. (1982) Grammaires floues, Pratiques, 33, 51-59, Metz.

CULIOLI, A. (1983) Pourquoi le français parlé est-il si peu étudié ?, Recherches sur le français parlé, 5, 163-207.

DAMOURETTE, J. et PICHON, E. (1911-1930) Des mots à la pensée. Essai de grammaire de la langue française, Tome Premier, Paris : D'Artrey.

DELATTRE, P. (1966) Les dix intonations de base du français, French Review, 40, 1-14.

DELAVEAU, A. et KERLEROUX, Fr. (1984) La constance de la raison linguistique, DRLAV, 31, 19-34. DELOMIER, D. et MOREL, M.-A. (1986) Caractéristiques intonatives des incises, DRLAV, 34-35, 141-160.

DERRIDA, J. (1967) De la grammatologie, Paris, Minuit.

DEULOFEU, J. (1979) Les énoncés à constituant lexical détaché. Les limites de l'organisation grammaticale et de l'organisation discursive dans les énoncés, Recherches sur le français parlé, 2 , 75-110.

DEULOFEU, J. (1981) Perspective linguistique et sociolinguistique dans l'étude des relatives en français, Recherches sur le français parlé, 3, 99-135. 
DEULOFEU, J. (1982) A propos des préjugés logicistes dans l'analyse grammaticale : le cas des prépositions. Hypothèses linguistiques et sociolinguistiques, Recherches sur le français parlé, 4 , $45-70$.

DEULOFEU, J. (1984) Sur les fondements empiriques de la recherche en syntaxe du français», LINX, 10, 57-74.

FORNEL (de), M. (1988) Constructions disloquées, mouvement thématique et organisation préférentielle dans la conversation, Langue française, 78, 101-123.

FREI, H. (1971) La grammaire des fautes (1929 pour la 1ère éd.), Genève, Slatkine.

GADET, Fr. (1991) Le parlé coulé dans l'écrit : le traitement du détachement par les grammairiens du XXe siècle, Langue Française, 89, 110-124.

GADET, Fr. et MAZIÈRE, Fr. (1986) Effets de langue orale, Langages, 81, 57-75.

GOODY, J. (1979) La raison graphique. La domestication de la pensée sauvage (1977 pour l'éd.

originale), Paris, Minuit.

GREVISSE, M. (1993) Le bon usage, 13e éd. refondue par A. GOOSSE, (1936 pour la 1ère éd.), Paris Louvain-la-Neuve : Duculot.

GUILHAUMOU, J. et MALDIDIER, D. (1984) Coordination et discours. Du pain et X à l'époque de la Révolution française, LINX, 10, 97-118.

HALLIDAY, M. (1990) Spoken and Written Language, F. CHRISTIES (éd.), (1ère éd. 1985), Oxford University Press.

HOUDEBINE, A.-M. (1979) Pour qui, pourquoi et comment transcrire ?, Le Français dans le monde, 145, 28-35.

JEANJEAN, C. (1982) Qu'est-ce que c'est que ça ? Étude syntaxique de ça sujet en français parlé : la construction quand $P+c ̧ a$, Recherches sur le français parlé, 4, 117-151.

MARTINET, A. (1965) Le mot, Diogène, 51, 39-53, art. reproduit dans MARTINET, 1975, Studies in Functional Syntax, Munchen, Wilhelm Fink Verlag (même pagination).

MERTENS, P. (1990) Intonation in BLANCHE-BENVENISTE Cl. et alii, Le français parlé - Études grammaticales, CNRS, Paris.

MOREL, M.-A. et RIALLAND, A. (1992) Emboîtements, autonomies, ruptures dans l'intonation française, Travaux linguistiques du CERLICO, 5, 221-243.

RUPPLI, M. (1991) Thématisation et coordination, Langages, 104, 46-61.

SAUSSURE (de), F. (1973) Cours de linguistique générale (1916 pour la 1ère éd.), Paris, Payot.

\section{ANNEXES}

\section{Protocole de transcription}

/ = rupture tonale

// = pause brève, /// pause plus longue, etc.

$/(\mathbf{r})$ = pause respiratoire, $/ /(r)$ pause respiratoire plus longue, etc. 
MAJUSCULES = syllabe accentuée de façon atypique (insistance, accent

didactique)

: = voyelle allongée

$<=$ intonation ascendante sur la fin du groupe rythmique

$\ll<$ intonation suraiguë

> = intonation descendante sur la fin du groupe rythmique

> = intonation conclusive

<> = intonation ascendante sur la syllabe pénultième, puis descendante

sur la dernière

$\wedge$ = liaison facultative réalisée

\# = liaison facultative non réalisée

Double soulignement= intonation "en sourdine" (parenthétique)

Simple soulignement $=$ prononciation d'un groupe consonantique

final chargé

(chev.) = chevauchement de syllabes

\section{NOTES}

1. À ce propos, voir l'article de Gadet et Mazière (1986), qui dressent l'inventaire des différents champs de recherche impliqués dans l'intrusion de données orales.

2. «Pour qui, pourquoi et comment transcrire?» s'interroge Houdebine (1979). Pour un panorama des problèmes posés par la transcription, cf. l'article de $\mathrm{S}$. Wachs dans le présent numéro.

3. On n'en finirait pas de dresser la liste de ceux qui ont dénoncé la discrétion des recherches linguistiques sur la langue parlée. Sur ce thème, l'appel de Culioli (1983) reste le plus célèbre.

4. Un peu plus tard (1929), on peut lire sous la plume de Frei qu'« en linguistique, toute vérité entre par les oreilles, toute sottise par les yeux» (p. 36 pour l'éd. de 1971) et, plus tard encore, Martinet écrit que «[...] c'est de l'énoncé oral qu'il faut toujours partir pour comprendre la nature réelle du langage humain» $(1965,51)$.

5. Pour une argumentation détaillée, cf. Derrida (1967, chap. III).

6. Car c'est bien de ce champ que l'oral se voit le plus exclu, ce que souligne Chervel $(1983,163$ sqq). Selon lui, au début du siècle, les linguistes ne s'intéressent que très peu à la syntaxe : à cette époque, l'étude des relations entre constituants est très largement confiée aux grammairiens, dont le normativisme débouche sur la mise à l'index de la langue parlée.

7. L'expression est d'Encrevé (in Bergounioux et alii, 1992, 102).

8. Une position qui paraît aujourd'hui minoritaire : l'idée - chère à Damourette et Pichon - d'un oral exploitant le système en le prolongeant a fait florès chez bon nombre de chercheurs contemporains. Par exemple, on la retrouve exploitée dans la notion de «supplétisme » chez Blanche-Benveniste (1983).

9. En l'occurrence, Halliday procède au décompte des mots grammaticaux et lexicaux.

10. Le débat en question a été diffusé simultanément sur France 2 et France Inter. Les conventions utilisées pour la transcription sont présentées en annexe. 
11. Numéros du 3 mai 1995.

12. Tout en étant conscient de l'inéluctable « trappe de la scription » dont parle Barthes $(1981,9)$. 13. En témoignent les contresens manifestes qu'on peut trouver, tel que [...] c'est compromettre leur milieu de carrière dans le Figaro, au lieu de [...] le: contremaître euh: / en milieu de carrière < // (corpus TOA).

14. Nous n'avons pu relever aucun choix de transcription imputable à la coloration politique de chacun des deux quotidiens : aussi cette piste a-t-elle été définitivement écartée, et l'utilisation du terme "idéologie" ne concerne pas cet aspect du problème.

15. Quelle qu'en soit leur forme, nous utiliserons pour l'instant le terme de "segmentation", qui paraît plus neutre que "thématisation" en particulier. Ce terme est utilisé par Bally pour désigner une des trois formes d'énonciation qu'il reconnaît, et qu'il définit comme «[...] une phrase unique issue de la condensation de deux coordonnées, mais où la soudure est imparfaite [...]» $(1965,60)$.

16. A ce sujet, cf. Gadet (1991).

17. Faute de place, nous ne les exposons pas toutes.

18. Cet effet de contraste créé par l'emploi de pronoms toniques est régulièrement évoqué dans la littérature : Danon-Boileau et alii parlent à ce sujet de «thème contrastif» $(1991,119)$. De Fornel souligne pour sa part que «l'apparition de constructions disloquées (...) permet de contraster des entités de discours » $(1988,115)$.

19. En l'occurrence, il s'agit du candidat du PS, qui avait réclamé un face-à-face dès avant le premier tour des élections : l'objet de l'énoncé, par conséquent, ne saurait être «Contrairement aux autres candidats, M. Chirac et moi organisons ce débat ».

20. Le terme est régulièrement utilisé pour ce type de construction : cf. par exemple Grevisse qui parle de « redondance expressive » (\$367b).

21. Coordonnants ou adverbes de liaison : principalement et, mais et alors.

22. C'est-à-dire $100 \%$ des 26 occurrences relevables dans les deux journaux.

23. Par exemple, dans une perspective conversationnelle, de Fornel (1988) propose une analyse des thèmes détachés non centrée sur les relations anaphoriques entre l'élément disloqué et le constituant de droite, mais entre celui-ci et ce qui précède.

24. L'article porte néanmoins sur des constructions spécifiques que nous n'exposerons pas ici.

25. La valeur intrinsèque de chaque connecteur introduisant un thème détaché aboutit à différents effets discursifs : "transition thématique » avec et, " thème contrasté " avec mais, etc.

26. Dans cette partie, on ne fera pas état de formes du type ah moi, ça va barder (oral spontané) où moi n'est pas repris à droite. Grevisse mentionne le cas de Moi, en général, c'est comme ça que ça se passe, et parle d'« anacoluthe » (\$228).

27. Neuf exemples dans chacun des deux quotidiens, soit dix-huit occurrences au total, dont quinze n'ont pas subi de modification.

28. Nous ne ferons ici qu'évoquer le faible prestige dont bénéficie ça, à quoi la norme préfère cela, sans qu'il s'agisse pour autant de pronoms de même distribution : par exemple, cela ne peut pas apparaître en (26), ni en disloqué, ni en terme de reprise. Pour d'autres arguments, cf. Jeanjean (1982).

29. Sauf en cas d'ambiguïté sur la coréférence : cf. Bonnard (1971, art. « Anacoluthe ») qui parle à ce sujet de «compléments mal attachés ", qu'il affecte d'ailleurs principalement à l'oral : «La langue admet que certains compléments soient séparés par une pause (que marque une virgule) et éventuellement éloignés, dans la chaîne orale, du terme auquel ils se rapportent (leur "support"). Encore faut-il qu'il n'en résulte aucune ambiguïté, et ce n'est pas le cas dans la phrase suivante : Souffrant du foie, je vous conseille d'éviter l'alcool » (nous soulignons).

30. Delomier et Morel (1986) signalent cependant qu'il est difficile de savoir ce qui détermine les phénomènes de reprise textuelle : les comptages auxquels elles ont procédé n'ont pas pu montrer 
le caractère déterminant de la longueur de l'incise. Cependant, lorsque le constituant précédant l'incise n'est pas autonome, elles signalent une plus grande fréquence de reprises textuelles.

31. Compte tenu de l'intonation descendante + pause après l'incise (cf. infra).

32. Titre donné par Grevisse au paragraphe 367 évoqué plus haut.

33. Pour aller vite, les dislocations peuvent être caractérisées par le phénomène intonatif de downstep, renvoyant à la différence de hauteur entre la fin du détaché et l'initiale du segment qui suit: à ce sujet, cf. Morel et Rialland, 1992. On peut également trouver une description prosodique des dislocations (principalement les DD) dans Ashby (1994).

34. A moins de poser une quelconque correspondance entre intonation et ponctuation, ce qui paraît peu envisageable.

35. A ce sujet, cf. Halliday (1990, 82 sqq) ainsi que Clément (1991).

36. Pour un inventaire plus détaillé de ces phénomènes, cf. Blanche-Benveniste et alii (1990, chap. III).

37. Deulofeu (1981) fait des remarques similaires à propos de certaines relatives non standard. Pour Berrendonner $(1990,32)$, le domaine des relations macrosyntaxiques aurait été dominant jusqu'en français classique.

38. Cf. par exemple Bosredon et Tamba (1992).

39. Nous utilisons ce terme à défaut d'un autre plus approprié, sans toutefois poser que ce type de phénomène soit nécessairement le résultat d'une procédure consciente.

40. Les recherches en phonostylistique et en "intonosyntaxe" s'accordent au moins sur cette caractérisation de la dépendance/autonomie syntaxique: de Delattre (1966) dégageant 10 « intonèmes " pour le français, dont deux contours ascendants de " continuation » jusqu'à Morel et Rialland (1992) pour qui «l'emboîtement» (grosso modo, la subordination) se produit en présence d'un ton final haut, en passant par Mertens (in Blanche-Benveniste et alii, 1990, 173) qui reconnait deux tons « infra-bas » (B-B- et HB圈) comme étant l'intonation d'autonomisation des énoncés.

41. Voir par exemple Goody (1979), qui apporte un éclairage ethnologique sur la question.

42. Pas plus qu'elle ne fait partie de ses ressources à proprement parler, même si la ponctuation rentre dans certains dispositifs tels que la juxtaposition.

43. Autrement dit, "Rendre à la structure ce qui est à la structure », pour reprendre la formule de Delaveau et Kerleroux $(1984,19)$.

\section{RÉSUMÉS}

Les formes linguistiques réputées les moins "prestigieuses" sont régulièrement mises sur le compte de l'usage parlé : une tendance souvent très présente dans l'esprit de ceux qui observent ou codifient la langue. Cela ne peut toutefois qu'entretenir la confusion faite entre le canal (oral $v s$ écrit) et l'actualisation de registres de style. Concevoir qu'une matérialité spécifique permet ou interdit certaines ressources paraît légitime, mais traitée trop rapidement, cette question débouche sur la confusion évoquée plus haut, surtout lorsqu'interfèrent certaines options socioculturelles prises sur la langue. Pour faire la part des choses, il convient donc d'envisager une comparaison systématique entre pratique orale et pratique écrite, ce que nous avons entrepris de faire par voie de "collation". 
The least prestigious linguistic patterns are often assigned to the spoken language by those who study or codify the language. Such a linking leads to perpetuate a confusion between the channel (spoken $v s$ written) and the actualization of style registers. Conceiving that a specific materiality allows or forbids any defined resources looks legitimate, but a too superficial examination induces us to the above-mentioned confusion, especially when sociocultural conceptions about the language interfere. In order to make allowances, it is fitting to proceed to a systematic comparison between spoken and written experiences, what we have undertaken through a work of "collation".

\section{AUTEUR}

\section{BRUNO MARTINIE}

